https://doi.org/10.7559/gestaoedesenvolvimento.2019.372

Data de receção: 29/01/2019 Data de aceitação: 23/07/2019

\title{
A GAMIFICAÇÃO NO ÂMBITO DOS SERVIÇOS: SCOPING REVIEW
}

\section{GAMIFICATION UNDER SERVICES: SCOPING REVIEW}

Margarida Afonso ${ }^{1}$ orcid.org/0000-0001-8567-8256 António Andrade ${ }^{2}$ orcid.org/0000-0001-8096-4720

Resumo: A gamificação, abordagem tecnológica inovadora, tem sido cada vez mais utilizada como forma de motivar a adoção de comportamentos benéficos nos utilizadores. A área do saber relativo à gamificação aplicada ao contexto dos serviços poderá traduzir-se em benefícios diversos - pessoais, sociais, organizacionais, entre outros e, como tal, importa proceder ao mapeamento sistemático e rigoroso da produção científica subjacente a este tema. Quanto ao método, partiu-se de uma abordagem de scoping review, analisando-se os dados através de duas aplicações - VOSViewer e Citespace - e partindo de métricas disponibilizadas pela Web of Science. Com base nisso realizou-se uma análise bibliométrica englobando vários parâmetros. Concluiu-se que a investigação científica sobre a gamificação e os serviços está ainda numa fase de desenvolvimento muito inicial. A análise realizada forneceu indicadores que sugerem que a gamificação se encontra numa trajetória de crescimento $e$ expansão no que respeita à sua aplicação no âmbito dos serviços, com particular ênfase nos serviços de saúde e de educação. A Finlândia parece ser o país que mais se destaca em termos de produção científica nesta área.

${ }^{1}$ Católica Porto Business School

${ }^{2}$ CEGE, Católica Porto Business School. Email: aandrade@ porto.ucp.pt 
Palavras-chave: Gamificação; Jogos; Serviços; Motivação; Saúde; Educação; Experiência; Finlândia; Bibliometria.

Abstract: Gamification, an innovative technological approach, has been increasingly used as a way to motivate the adoption of beneficial behaviors in users. The area of knowledge related to gamification applied to the context of services may translate into diverse benefits personal, social, organizational, among others - and, as such, it is necessary to systematically and rigorously map the scientific production underlying this theme. As for the method, this article was based on a scoping review approach, analyzing the data through two applications - VOSViewer and Citespace - and starting from metrics made available by the Web of Science. Based on this, a bibliometric analysis encompassing several parameters was performed. It was concluded that scientific research on gamification and services is still at a very early stage of development. The analysis provided indicators that suggest that gamification is on a growth and expansion trajectory with respect to its application in services, with particular emphasis on health and education services. Finland seems to be the country that stands out most in terms of scientific production in this area.

Keywords: Gamification, Games, Services, Motivation, Health, Education, Experience, Finland, Bibliometrics.

\section{INTRODUÇÃO}

Nos anos recentes temos assistido ao surgimento e expansão daquilo que se designa por gamificação (Hamari, 2013), uma abordagem tecnológica inovadora que procura motivar as pessoas para a realização de atividades, tarefas e comportamentos benéficos (no plano individual e social) (Hamari \& Koivisto, 2015a, 2015b).

A gamificação tem sido aplicada em diversos domínios - e.g. educação, comércio, comunicação e atividades intra-organizacionais, serviços governamentais, comportamento ambiental, consumo sustentável, marketing e publicidade, saúde, estilo de vida e bem-estar (Hamari \& Koivisto, 2015a; Koivisto \& Hamari, 2014). Na base disto está 
a ideia de que "como os jogos são divertidos, qualquer serviço que use mecanismos idênticos deverá também ter mais valor e ser mais envolvente" (Hamari, 2013, p. 236).

\section{BACKGROUND}

A gamificação está associada a tecnologias que procuram promover motivações intrínsecas relativamente a várias atividades e tarefas em contextos non-gaming, normalmente, através do uso de caraterísticas e mecanismos dos jogos (Hamari \& Koivisto, 2015a; Ueyama, Tamai, Arakawa, \& Yasumoto, 2014). Na perspetiva de Hamari and Koivisto (2015a), é uma "abordagem tecnológica emergente para motivar as pessoas em direção a diferentes tipos de comportamentos benéficos" (Hamari and Koivisto, 2015a, p. 333). Essa motivação ocorre através da "restruturação dos objetivos de longo-prazo ao fornecer aos utilizadores objetivos de curto-prazo, atividades, recompensas e apoio social" (Hamari \& Koivisto, 2015a, p. 333). Procura-se proporcionar experiências semelhantes às existentes nos jogos, tornando as atividades mais envolventes (Deterding et al., 2011, in Koivisto \& Hamari, 2014). Num contexto aceleradamente tecnológico e com a chegada de jovens às organizações, quer como colaboradores, ou como clientes, que cresceram com jogos digitais, emerge claramente a gamificação como uma estratégia particularmente adequada em contexto organizacional. Serve dimensões como a motivação dos colaboradores, a sua capacidade de comunicação interna e externa e o desenvolvimento de espírito de equipa, como competências tão fundamentais ao sucesso organizacional (Almeida, Capucho, \& Ribeiro, 2017).

Exemplos de elementos de jogos típicos na gamificação são as pontuações, quadros de ranking, conquistas, feedback, objetivos claros e narrativa (Hamari \& Koivisto, 2015a). Koivisto and Hamari (2014) distinguem os conceitos de gamificação e de jogo - enquanto o primeiro é, normalmente, usado para promover objetivos externos ao jogo (e.g. estilos de vida saudáveis, consumo sustentável), a participação em jogos associa-se a motivações puramente intrínsecas.

A gamificação pode ser definida de uma perspetiva sistémica, em que a adição e utilização de mecanismos e elementos de jogo em contextos 
non-game tem o potencial de transformar os serviços em jogos (Deterding et al., 2011, cit in Hamari, 2013, p. 237). Já numa perspetiva experiencial a gamificação é vista como "um processo que proporciona oportunidades de experiências de jogos e que suporta a criação de valor global pelos clientes" (Huotari \& Hamari, 2012, cit in Hamari, 2013, p. 237); aqui, é o cliente que, em última instância, decide se a experiência de jogo proporcionada aumenta o valor do serviço ou não. Daqui decorre que a mera adição de mecanismos de jogos a um serviço não leva automaticamente à maior envolvência do serviço e maior retenção de clientes; para a gamificação ter sucesso é necessário que os consumidores estejam previamente envolvidos em experiências de jogos (Hamari, 2013).

\section{ABORDAGEM METODOLÓGICA}

A análise da produção científica é um indicador útil para compreender o estado atual de uma determinada área de estudo.

Esta Scoping Review baseou-se no método descrito por Arksey and O'Malley (2005) que inclui: 1) identificação da questão de investigação, 2) identificação dos estudos relevantes, 3) seleção dos estudos, 4) mapeamento dos dados e, por fim, 5) agrupar, sumariar e reportar os resultados. Foi adotada uma perspetiva temporal para analisar e visualizar de forma sistemática e global a extensão, abrangência e natureza da investigação científica subordinada à gamificação nos serviços.

$\mathrm{Na}$ análise dos dados utilizou-se a bibliometria - "técnica quantitativa e estatística que permite medir índices de produção e disseminação do conhecimento, acompanhar o desenvolvimento de diversas áreas científicas e os padrões de autoria, publicação e uso dos resultados de investigação" (Araújo, 2006, cit in Lopes, Costa, Fernández-Llimós, Amante, \& Lopes, 2012, p. 2). Os indicadores bibliométricos permitem avaliar a situação atual da produção científica numa determinada área e o seu estado atual de desenvolvimento (Liao et al., 2018; Lopes et al., 2012). Embora haja uma multiplicidade de indicadores (de qualidade científica, de atividade científica, de impacto e de associações temáticas) (Lopes et al., 2012), neste estudo apenas se utilizaram indicadores de atividade científica e indicadores de impacto científico. 


\subsection{Identificação dos estudos relevantes}

Para identificar os estudos relevantes procedeu-se à pesquisa na base de dados Web of Science nos dias 10 e 11 de abril de 2018. Definiram-se critérios com vista à extração dos estudos relevantes. Procedeu-se à combinação de duas palavras-chave: TS = ("Gamification") AND ("Services").

A pesquisa foi limitada pelo uso de filtros. Inicialmente foi definido como critério de inclusão o período temporal de 2008 a 2018, de modo a analisar os últimos dez anos de atividade científica e a obter uma perspetiva abrangente. No entanto, a primeira publicação subordinada ao tema com as palavras-chave acima mencionadas surgiu em 2011, indicando o estado embrionário deste tópico. Por essa razão, encurtou-se o intervalo de análise e foram incluídos todos os estudos publicados entre 2011 e 2018 (últimos oito anos). Não obstante, o conceito de jogo aplicado à formação e à educação é muito antigo. Pode ter antecedentes milenares no jogo de xadrez até suscitar um grande, renovado e ininterrupto interesse, a partir da década de sessenta do século passado (Lopes \& Andrade, 2008).

Quanto ao idioma, apenas se incluíram artigos publicados em Inglês. Foram incluídos os estudos independentemente da arbitragem científica (peer-review) e de serem de âmbito nacional ou internacional.

\subsection{Seleção dos estudos}

Os artigos extraídos foram incluídos na medida em que se dirigiam, simultaneamente, a tópicos relacionados com a "Gamification" e "Services", independentemente destes termos se encontrarem no título, abstract, palavras-chave ou em qualquer outra parte do artigo.

Como resultado da pesquisa realizada foram obtidas 195 publicações científicas que cumpriam os critérios de seleção. Ao total de publicações correspondiam quatro tipos de documentos, sendo o mais frequente 'Proceedings papers' (109), seguindo-se o 'Article' (81), "Review' (5) e "Editorial Material' (2). Todos os documentos foram descarregados no dia 11 de abril de 2018 no formato 'Separado por tabulações'.

Tendo em conta que esta é uma análise abrangente do tema gamificação nos serviços, e tendo sido utilizada uma única base de dados eletrónica, o risco de haver repetição de artigos é nulo e, como tal, não se 
considerou necessária a realização de screening. Deste modo, foram consideradas para análise a totalidade das publicações científicas.

\section{REALIZAÇÃO DA SCOPING REVIEW}

\subsection{Questões de investigação}

O objetivo deste estudo consistiu na análise rigorosa e sistemática da produção científica no âmbito da gamificação nos serviços. Considerouse como 'produção científica' os artigos publicados em revistas científicas (Arksey \& O'Malley, 2005).

Definiu-se a seguinte questão de investigação: Qual a evolução da produção científica no estudo da gamificação no âmbito dos serviços? Emerge desta questão o objetivo de identificar as redes de investigação, a sua geografia e quais as dimensões do conceito mais estudadas.

\subsection{Mapeamento dos dados}

Os dados referentes às publicações foram extraídos e mapeados de modo à realização da análise bibliométrica, através do VOSviewer e Citespace. O primeiro foi usado para visualização de mapas de redes de co-autoria e redes de co-citações; o Citespace permitiu a visualização cronológica de palavras-chave. Para complementar, foram também usadas métricas e indicadores disponibilizados pela Web of Science.

Foi com base nos mapas obtidos das aplicações que se procedeu à análise dos indicadores bibliométricos - de atividade científica e de impacto científico. O primeiro indicador permite "contabilizar a atividade científica desenvolvida, nomeadamente o número e distribuição dos trabalhos publicados, a produtividade dos autores, a colaboração na autoria dos trabalhos, o número e distribuição das referências entre trabalhos e autores" (Lopes et al., 2012, p. 2). "Os indicadores de impacto científico podem subdividir-se em dois tipos: indicadores de impacto dos trabalhos e indicadores de impacto das fontes" (idem, p. 2). O primeiro exemplifica-se através do número de citações recebidas; já o segundo tem como exemplo o "fator de impacto das revistas, o índice de citação imediata e a influência das revistas" (idem, p. 2). 


\section{RESULTADOS}

Os resultados são apresentados em quatro secções. O estado atual do estudo da gamificação nos serviços, a análise das palavras-chave dos hotsposts de investigação e, finalmente, apresentam-se as análises da coautoria e da co-citação.

\subsection{Estado atual do estudo da gamificação nos serviços}

1) Tendências anuais das publicações científicas

O Gráfico I demonstra a tendência temporal das publicações científicas. Desde o primeiro artigo publicado em 2011, tem havido um padrão global de crescimento da atividade científica nesta área, embora tenha ocorrido um ligeiro decréscimo em 2017. Tendo em conta que estamos no início de 2018 compreende-se a discrepância de números, sendo expectável um aumento de publicações ao longo deste ano. O padrão de crescimento positivo parece indicar que desde 2011 tem havido um interesse cada vez maior dos investigadores acerca do tema.

\section{Gráfico I}

Evolução temporal de publicações científicas (2011-2018)

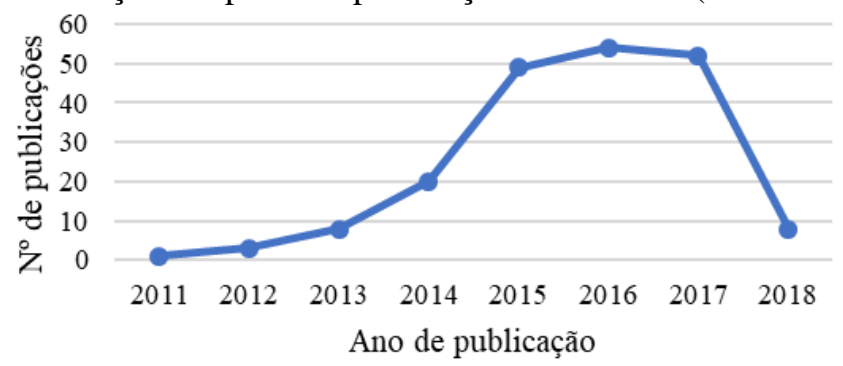

2) Distribuição das organizações e dos países

A Universidade de Tampere (Finlândia) tem o maior número de publicações (10 papers; 5,13\% do total de publicações) - Gráfico II. Na segunda posição encontra-se o Instituto de Tecnologia de Bandung (Indonésia) com 9 papers publicados (4,62\%), seguindo-se a Aalto 
University (Finlândia) com 8 papers $(4,10 \%$ ), e a Waseda University (Japão) com 4 (2,05\%). Verifica-se a diversidade de países nos quais tem existido atividade científica nesta área de investigação, sendo a Finlândia aquele com maior prevalência, com três institutos no top 10. Em geral, oito das dez organizações são europeias, sendo as outras duas asiáticas. Os dados sugerem uma grande atividade científica na Europa comparativamente às restantes regiões

\section{Gráfico II}

Top 10 de Organizações com publicações de gamificação e serviços

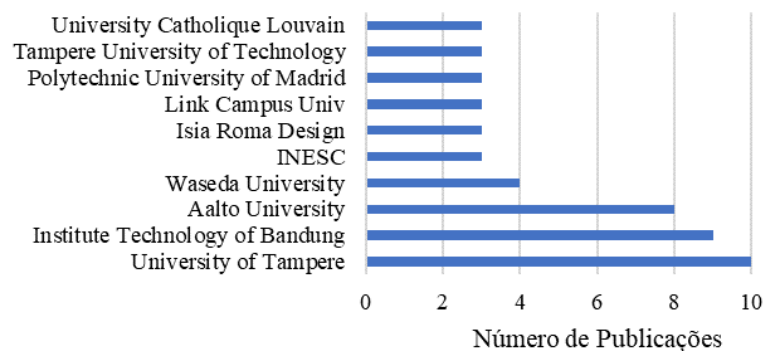

3) Análise de citações e do h-index por país

Utilizou-se como indicador da qualidade (de artigos, autores, organizações) a frequência de citações recebidas - quanto mais é citado relativamente a outros, maior a qualidade (Bornmann et al., 2012, in Tahamtan, Afshar, \& Ahamdzadeh, 2016).

As 195 publicações foram citadas 607 vezes. O número de citações da Finlândia foi 431 - Gráfico III -, correspondendo a 71,0\% do total de citações, seguindo-se o Japão com 57 citações $(9,39 \%$ da totalidade das citações). 


\section{Gráfico III}

Soma de citações por país.

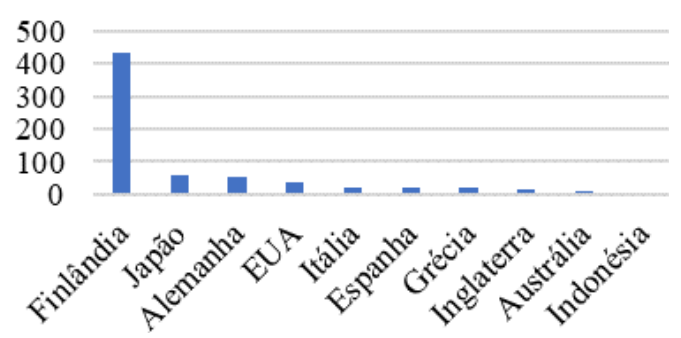

Utilizou-se o parâmetro h-index que quantifica as conquistas científicas (Liao et al., 2018) e combina medidas de quantidade (publicações) e de impacto (citações), aplicando-se a investigadores, organizações e/ou países (Lopes et al., 2012). Permite "criar rankings de universidades, analisando e comparando universidades produtivas e de baixo impacto versus universidades menos produtivas e de maior impacto" (idem, p. 3). O índice "corresponde ao número de artigos de um determinado autor com, pelo menos, o mesmo número de citações" (Costas et al., 2007, cit in Lopes et al., 2012, p. 3).

Através do Gráfico IV verifica-se que é a Finlândia que tem o maior h-index (de 9), seguindo-se os EUA com 5, e a Alemanha com 4.

\section{Gráfico IV}

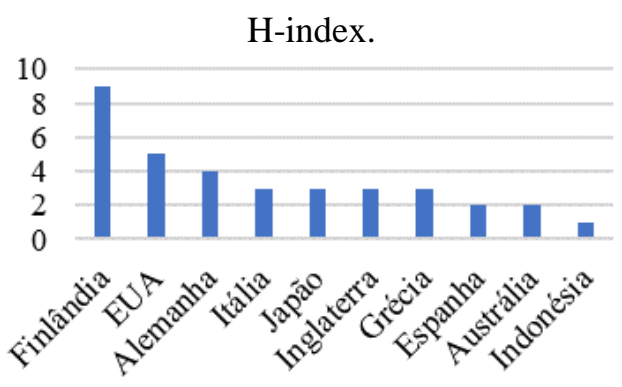

Relativamente ao Gráfico V, é possível verificar que o país líder no que respeita ao número de publicações é a Finlândia, seguindo-se Espanha (20). 


\section{Gráfico V}

Número de publicações por país.

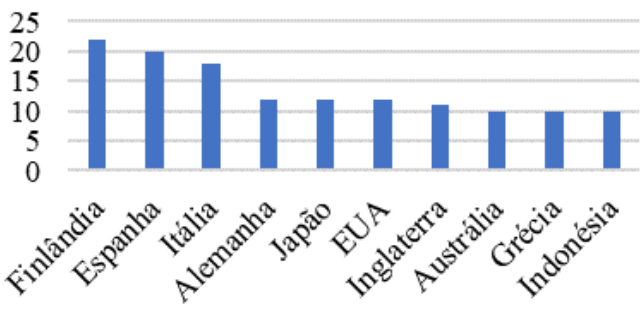

Analisando os Gráficos III, IV e V em conjunto conclui-se que a Finlândia é o país que se mantém sempre em primeiro lugar, tendo o maior número de citações, o maior h-index e a maior quantidade de publicações. Parece, pois, ser aquele com maior produção e impacto científico. Já a Espanha, embora esteja em segundo lugar no número de publicações (Gráfico V) -, na frequência de citações e no h-index encontra-se em sexto e oitavo lugar, respetivamente. Isto poderá indicar que a qualidade das publicações em Espanha precisa de melhorar, comparativamente aos restantes países. No caso dos EUA verifica-se que, embora não seja dos países com maior produção científica, é o segundo país em que a atividade científica subjacente ao tema tem maior impacto, com um h-index de 5.

4) Análise de citações e do h-index por investigador

No Gráfico VI é possível identificar Hamari J. como o autor com o maior número que publicações (11), seguindo-se Raflesia SP. e Surendro K. com cinco publicações cada um.

\section{Gráfico VI}

Número de publicações por autor.

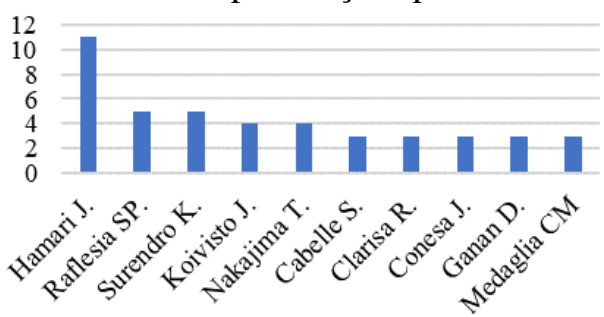


Com base no Gráfico VII, observa-se a soma de citações por autor, assumindo posições de destaque três autores: Hamari J. (403), Koivisto J. (174) e Nakajima T. (25).

\section{Gráfico VII}

Soma de citações por autor

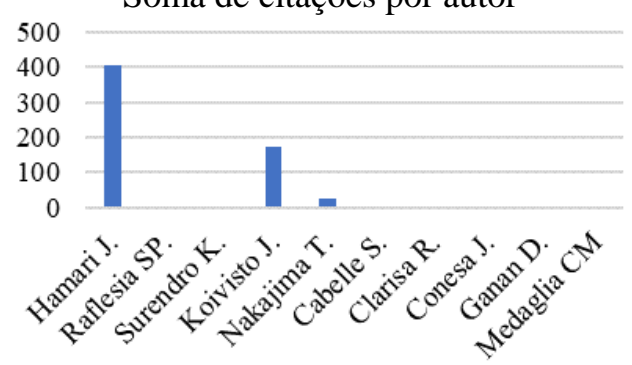

O Gráfico VIII demonstra o h-index dos diversos autores, sendo possível observar a relevância de Hamari J., com o h-index de 8. Isto significa que, da totalidade publicações científicas, o autor tem 8 artigos em que, cada um deles, tem pelo menos 8 citações. Releva mencionar os autores Koivisto J. e Nakajima T. com h-index de 4 e 2, respetivamente.

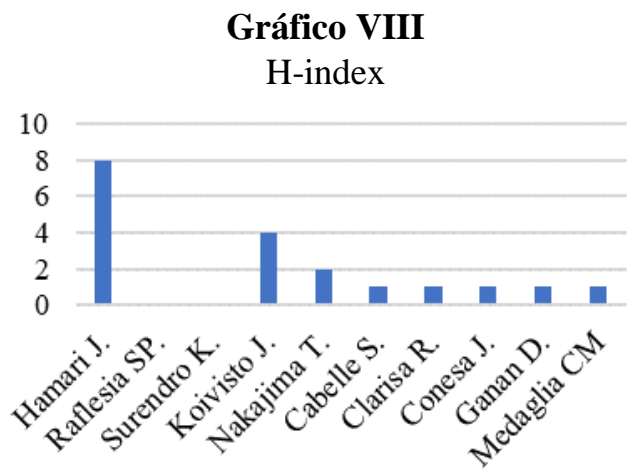

Analisando comparativamente os Gráficos VI, VII e VIII, destaca-se a relevância de Hamari J. nas três vertentes (número de publicações, soma de citações e h-index). Pelo contrário, por exemplo, Raflesia SP. e Surendro K., embora estejam bem posicionados em termos de número de 
publicações, verifica-se que não têm qualquer citação, e o h-index de cada um é 0; ou seja, apesar de terem artigos científicos publicados, na realidade não assumem uma posição central nesta área de estudo. Já, Koivisto J. e Nakajima T. apresentam maior relevância na medida em que apesar de terem um menor número de publicações científicas, têm uma grande quantidade de citações e apresentam h-index elevados (em relação aos restantes autores).

\subsection{Análise das palavras-chave}

\section{1) Análise da co-ocorrência de palavras-chave}

Esta análise procurou identificar os pontos nucleares de pesquisa acerca da gamificação e serviços. Das 195 publicações resultaram 38 palavras-chave, a partir das quais se obteve a rede de co-ocorrência de palavras-chave na Figura I. O tamanho de cada ponto representa o seu peso - quanto maior for, maior peso a palavra-chave terá. A distância entre dois pontos representa a força da relação - quanto menor a distância maior a força da relação. A linha entre dois pontos significa que apareceram simultaneamente, sendo que quanto maior a sua espessura, mais coocorrências terão havido entre ambos.

\section{Figura I}

Rede de co-ocorrência de palavras-chave

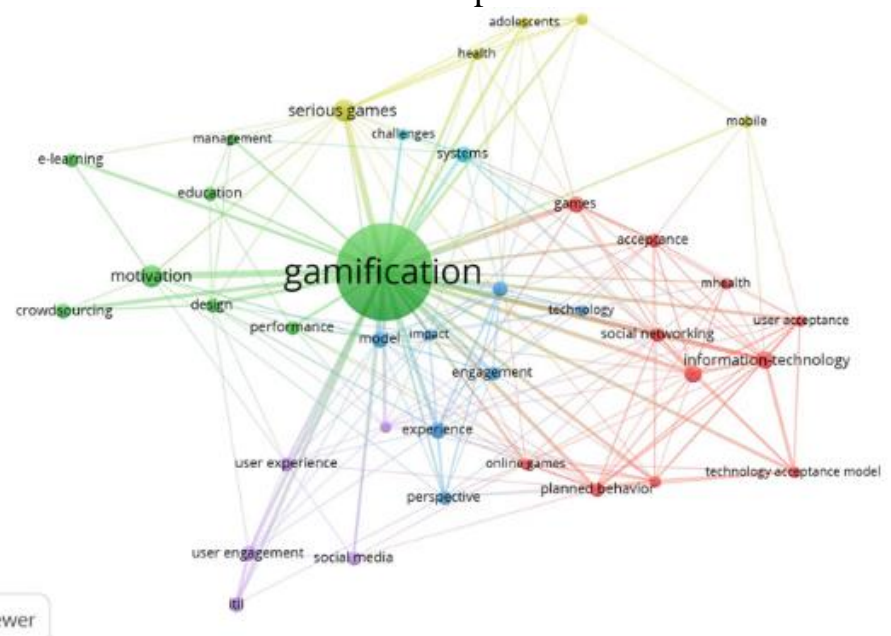


No Quadro I estão as 10 palavras-chave com maior frequência e a sua total link strength. A link strength entre dois pontos corresponde à frequência da co-ocorrência e funciona como indicador quantitativo para representar a relação entre esses pontos. A total link strength de um ponto é a soma das link strengths desse ponto relativamente aos restantes.

\section{Quadro I}

Top 10 de palavras-chave

\begin{tabular}{cccc}
\hline Rank & Palavras-chave & Frequência & Total Link Strength \\
\hline 1 & Gamification & 131 & 185 \\
2 & Serious games & 13 & 31 \\
3 & Information-technology & 9 & 39 \\
4 & Intrinsic motivation & 9 & 35 \\
5 & Experience & 8 & 27 \\
6 & Planned behavior & 6 & 30 \\
7 & Social networking & 6 & 26 \\
8 & Acceptance & 6 & 21 \\
9 & Persuasive technology & 5 & 23 \\
10 & User acceptance & 5 & 22
\end{tabular}

O ponto "gamification" tem ligações mais espessas com "informationtechnology" (39), "intrinsic motivation" (35), "serious games" (31), "planned behavior" (30), "experience" (27), "social networking" (26), "persuasive technology" (23), "user acceptance" (23) e "acceptance" (22).

As ligações entre "gamification" e "e-learning", "education", "management", "motivation" e "performance" sugerem que a gamificação tem sido estudada e aplicada nos serviços de educação, provavelmente como forma de aumentar a motivação e o desempenho dos utilizadores (estudantes). Por exemplo, Yamabe \& Nakajima (2013) estudaram o contexto educativo procurando analisar os benefícios da gamificação no processo de aprendizagem.

As relações entre "gamification" e "adolescents", "children", "health", "mobile", e "serious games" poderá significar que a gamificação também é estudada nos serviços de saúde, direcionando-se em concreto a jovens e adolescentes, visando a promoção de estilos de vida saudáveis. De facto, Ahola et al. (2013) analisaram a gamificação e o seu potencial no incentivo à atividade física e promoção de estilos de vida saudáveis para jovens do sexo masculino. 
As relações entre "gamification" e "engagement", "experience", "impact", "innovation", "model", "perspective", "technology" é um indicador de que a gamificação, enquanto tecnologia inovadora, é aplicada maioritariamente como estratégia para promover o envolvimento de um público-alvo, influenciando a sua experiência num certo contexto. As relações entre "gamification" e "game design", "social media", "user engagement" e "user experience" poderá refletir o facto da gamificação ser implementada como forma de aumentar o comprometimento e o envolvimento do utilizador com um determinado serviço/contexto/entidade, afetando a sua experiência. As relações entre "gamification" e "acceptance", "information-technology", "intrinsic motivation", "mhealth", "persuasive technology", "planned behavior", "social networking", "technology acceptance model" e "user acceptance" podem significar que a gamificação tem surgido no âmbito das tecnologias de informação para promover a motivação intrínseca do utilizador e planear certos comportamentos em contexto de redes sociais mediadas. Parece, também, que o sucesso desta tecnnologia inovadora depende da aceitação do utilizador e, por isso, é compreensível que, em termos de racional teórico, surja o modelo da aceitação da tecnologia.

Por fim, as relações entre "gamification" e "challenges" e "systems", reflete que a gamificação é ainda uma ferramenta bastante recente e, como tal, a sua aplicação a diversos sistemas parece constituir um desafio atualmente.

O software VOSviewer também permite visualizar a densidade da rede - Figura II. Destaca-se o ponto gamification a amarelo que, relativamente aos restantes, apresenta maior densidade (aparece mais frequentemente). Todos os outros pontos, ao aparecerem numa cor mais próxima do verde e azul indicam que não aparecem tantas vezes. Esta análise é útil para saber quais as áreas mais importantes num mapa de conceito (Liao et al., 2018). Neste caso, o mapa não apresenta uma grande variedade de cores, visto que se baseou numa amostra relativamente pequena de publicações cientificas, impedindo a extração de resultados significativos a este nível. Tal como expectável, a gamificação assumiu grande relevância ao ser a palavra-chave mais densa no âmbito das publicações científicas selecionadas. 


\section{Figura II}

Mapa de densidade de palavras-chave

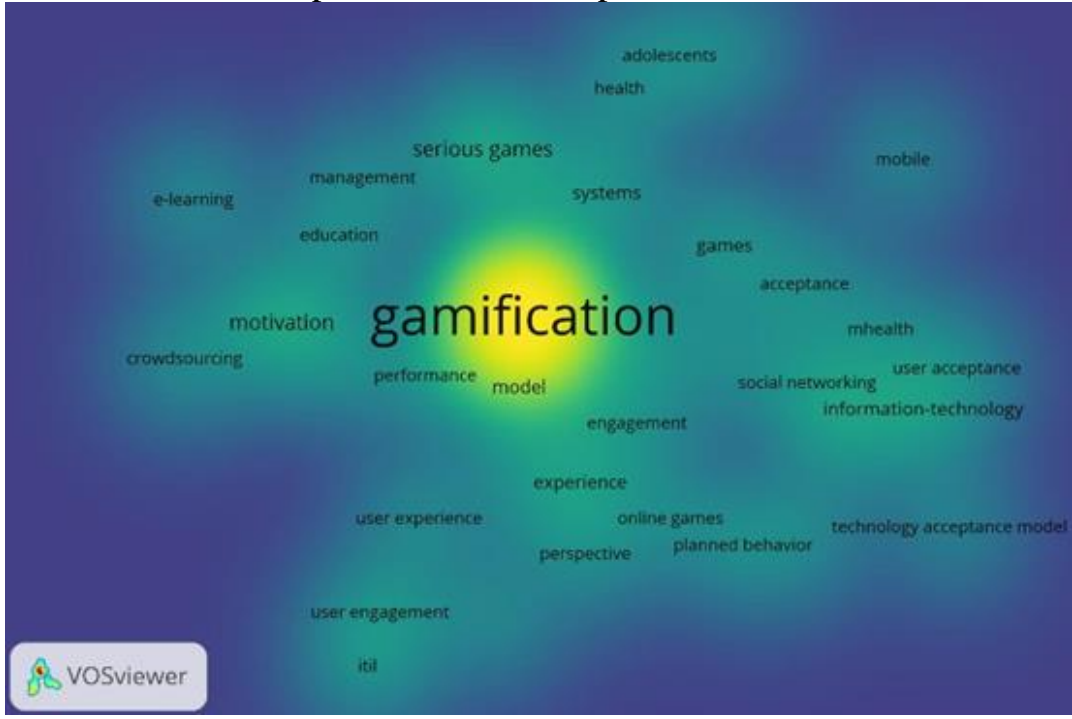

\section{2) Timeline de palavras-chave}

Através do software Citespace foi possível visualizar as principais palavras-chave numa perspetiva temporal - Figura III - bem como a evolução dos vários clusters de palavras-chave ao longo do tempo Quadro II. Através da Figura III não é possível identificar um padrão evidente entre 2011 e 2018. Ainda assim, no Quadro II verifica-se que, aquando o surgimento desta área de estudo, os autores começaram por analisar a gamificação na perspetiva de influência social, sendo considerada uma tecnologia persuasiva. Desde logo começou a ser implementada no contexto de serviços de saúde, sendo aplicada, um pouco mais tarde a serviços de educação, bancários, e enquanto estratégia para integrar os clientes e motivar os trabalhadores.

Com base na Figura III verifica-se que a partir de 2017, o estudo da gamificação nos serviços tem vindo a alargar o seu âmbito, ocorrendo uma expansão em termos de perspetivas de investigação. Além disso, sobretudo até 2013, há uma quantidade reduzida de investigação, 
começando em 2014 a surgir uma maior diversidade e extensão de palavras-chave e clusters.

Ainda assim, e de forma global, a gamificação tem vindo a ser estudada no âmbito de dois serviços principais que, ao longo dos anos, têm assumido particular centralidade e que, por isso, se destacam. São eles os serviços de saúde e os serviços de educação.

\section{Figura III}

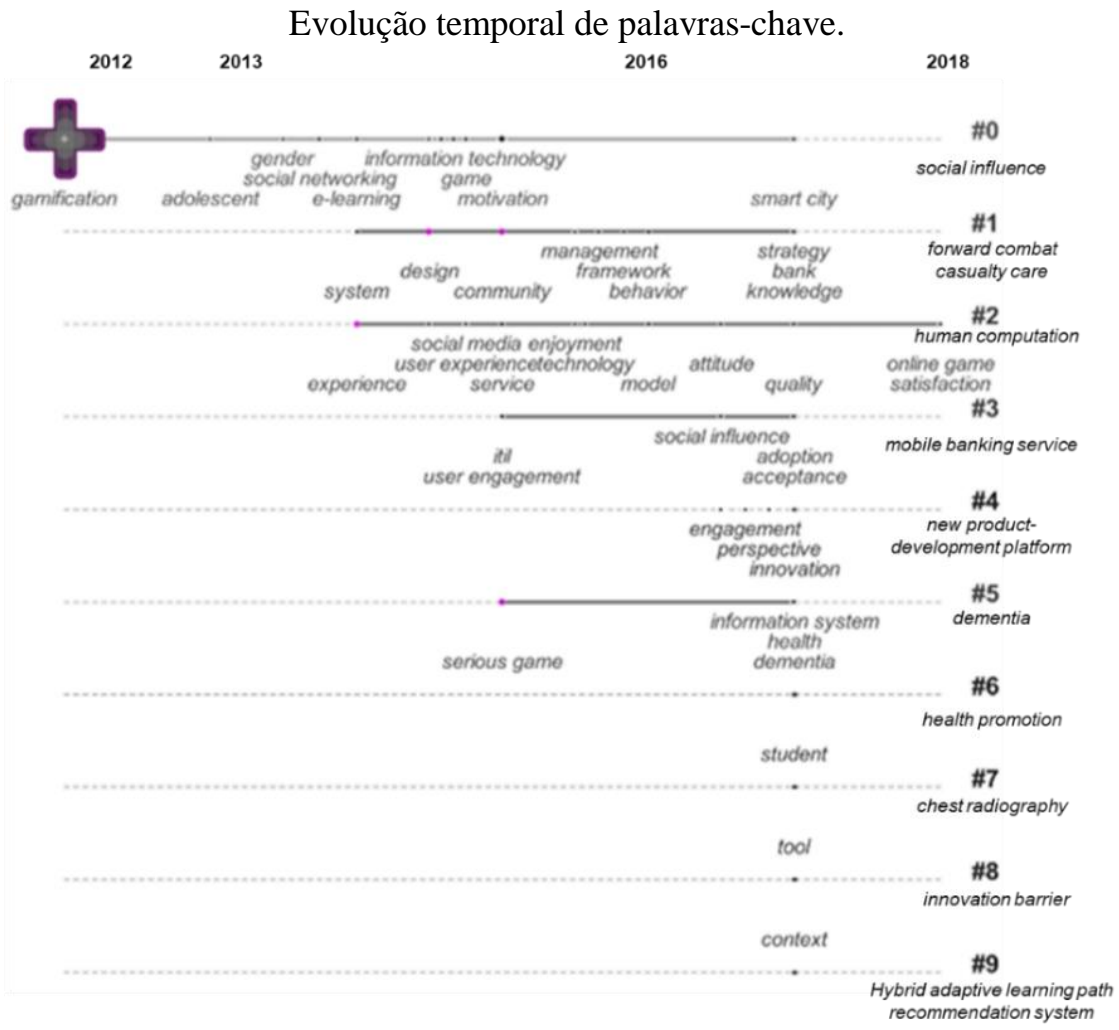




\section{Quadro II}

\section{Palavras-chave de publicações científicas e respetivos clusters numa perspetiva temporal.}

\begin{tabular}{|c|c|c|}
\hline Ano & Keywords & Cluster \\
\hline 2012 & $\begin{array}{l}\text { Service industry; persuasive technology; serious games; human } \\
\text { computer interaction }\end{array}$ & Social influence \\
\hline 2013 & $\begin{array}{l}\text { Social network; transtheoretical model; healthcare compliance; } \\
\text { service design; effectiveness }\end{array}$ & Social influence \\
\hline \multirow{3}{*}{2014} & Business training; game; persuasive technology; health & Social influence \\
\hline & Data; interactive water services; fault detection; student motivation & $\begin{array}{l}\text { Forward combat } \\
\text { casualty care }\end{array}$ \\
\hline & Games; health; experience & Human Computation \\
\hline \multirow{4}{*}{2015} & $\begin{array}{l}\text { Interactive learning; active learning; customer integration; work } \\
\text { motivation; multiple regression model }\end{array}$ & Social influence \\
\hline & $\begin{array}{l}\text { Edutainment; pervasive learning; pervasive serious games; empirical } \\
\text { studies; user studies; collaborative learning }\end{array}$ & $\begin{array}{l}\text { Forward combat } \\
\text { casualty care }\end{array}$ \\
\hline & $\begin{array}{l}\text { Student engagement; massive open online course; user experience; } \\
\text { computer games; behaviour psychology }\end{array}$ & Human Computation \\
\hline & User engagement; service support; service desk & $\begin{array}{l}\text { Mobile banking } \\
\text { service }\end{array}$ \\
\hline
\end{tabular}

Serious games; augmented reality games; edutainment; mixed reality; Dementia pervasive learning

Game-based learning; building services engineering; fire; serious $\quad$ Forward combat games; interdisciplinary collaboration

casualty care

2016

Stem education; intelligent tutoring systems; elearning platform;

Human Computation

software development framework; software analysis

Computing; mobile computing; crowd-sensing; smart cities; daily life Social influence

issues; mobile computing

Advanced-service transformation; servitization; student engagement; Forward combat researchers; students

casualty care

Transformative service research; information system; behavioral intentions; hedonic feature; user experience

Human Computation

Mobile app healthcare; sustained maintenance; digital rhetoric; social Mobile banking influence; social infrastructure

service

Online platforms; new product development; game design; service

design; servicemarketing

New product-

development

platform

2017 Augmented reality; we-intention; public helth; transmitted infections; Dementia young adults

Intelligent learning recommender system; mathematics education; Health promotion learning style; health promotion; subjective wellbeing

Social networking; self-management; resident education; social media Chest radiography

Value proposition; innovation barrier; health care; smart technology Innovation barrier

Game design; persuasive technologies; location-based games; weintention; online games

Hybrid adaptive

learning path

recommendation

system

2018 Social marketing; game design elements; motivational information

Human Computation system; quantified-self; social networking 


\subsection{Análise da co-autoria}

Uma parte importante da bibliometria consiste na análise da coautoria. É frequente os projetos de investigação serem realizados entre vários autores de forma colaborativa - "o nível de colaboração constitui um índice para avaliar o estado atual da investigação numa área em específico" (Bates et al., 2014, cit in Liao et al., 2018, p. 9). Nesta secção analisar-se-á a co-autoria por país e por organização, bem como as publicações mais citadas.

\section{1) Análise da co-autoria por país}

Analisa-se aqui o grau de comunicação entre países, e identifica-se os mais influentes no estudo da gamificação e serviços - Figura IV. Quanto maior o node maior a influência do país para o estudo desta área. As relações entre eles refletem a cooperação existente entre os institutos (Liao et al., 2018).

\section{Figura IV}

Rede de co-autoria de países de publicações científicas

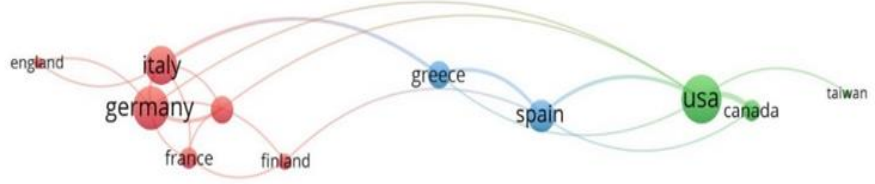

Com base na Figura IV e no Quadro III observa-se que os EUA, embora não sejam o país com maior número de artigos publicados (12) e citações (35), são o país com a maior total link strenght (9); quer isto dizer que tem o maior número de relações colaborativas com os restantes países - Alemanha, Espanha, Grécia, Portugal, Canadá e Taiwan. Já a Finlândia, apesar de ser aquele que contabiliza o maior número de publicações (22) e citações (430), tem uma total link strenght de 3 - ligações colaborativas a Espanha, Alemanha e França -, consideravelmente menor comparativamente aos EUA. 


\begin{tabular}{cccc}
\multicolumn{4}{c}{ Quadro III } \\
Co-autoria de países. \\
\hline País & Artigos & Citações & Total link strength \\
\hline EUA & 12 & 35 & 9 \\
Alemanha & 12 & 52 & 8 \\
Itália & 18 & 20 & 7 \\
$(\ldots)$ & $(\ldots)$ & $(\ldots)$ & $(\ldots)$ \\
Finlândia & 22 & 430 & 3 \\
\hline
\end{tabular}

Analisando a link strenght, identifica-se uma tendência para haver mais relações colaborativas entre países geograficamente próximos. Por exemplo, a intensidade das ligações entre os EUA-Canadá é de 3, e entre Itália-Grécia, Portugal-Alemanha, e Grécia-Espanha é de 2. Pelo contrário, as ligações colaborativas entre EUA-Alemanha, EUA-Portugal e EUA-Taiwan têm cada uma a força de 1. Isto não significa que não haja exceções, como é o caso da ligação entre EUA-Espanha com uma força de 2; ou seja, é provável que a proximidade geográfica constitua um fator influenciador das relações colaborativas entre os institutos presentes em cada país.

\section{2) Análise da co-autoria por organizações}

Nesta análise o VOSviewer apenas identificou três institutos, havendo uma única ligação entre dois deles - Quadro IV. A University of Tampere (Finlândia) é a organização com mais artigos publicados, mais citações e com uma total link strength de 5 - demonstra um grau elevado de relações colaborativas com as outras organizações. Ao mesmo nível encontra-se a Alto University (também na Finlândia), mas com menos artigos e citações.

\section{Quadro IV}

Co-autoria de organizações

\begin{tabular}{cccc}
\hline Organização & Artigos & Citações & Total link strength \\
\hline Aalto Univ & 7 & 165 & 5 \\
Univ Tampere & 10 & 290 & 5 \\
Bandung Inst Technol & 7 & 0 & 0
\end{tabular}




\section{3) Publicações científicas mais citadas}

Procedeu-se à identificação dos papers mais influentes na área da gamificação e serviços. No Quadro V apresenta-se as dez publicações científicas com maior número de citações, bem como a respetiva revista, autores e ano. Os mais citados foram publicados entre 2013 e 2016. A publicação científica com maior número de citações até ao momento é de J. Hamari (2013) com 108 citações, tal como se pode verificar pela dimensão e centralidade do ponto correspondente na Figura V. Cinco das dez publicações mais citadas tiveram, entre outros autores, a colaboração de J. Hamari, o que revela a sua centralidade no estudo deste tema.

O artigo mais citado - Hamari (2013) - estudou os efeitos da gamificação na retenção do utilizador. Concluiu que os utilizadores de um serviço gamificado e que assumiram uma postura ativa relativamente aos emblemas, tinham "uma probabilidade significativamente maior de usar ativamente o serviço" (Hamari, 2013, p. 243). Segundo o autor, "poderá ser mais fácil demonstrar a eficácia da gamificação em ambientes que atraem uma população de utilizadores já recetiva a interações com jogos" (idem, p. 244).

Uma das principais referências no âmbito da gamificação é a utilização de emblemas (badges) - o blueprint da gamificação - como forma de recompensar os usuários pela realização de um determinado comportamento (Hamari, 2013). Os emblemas são um mecanismo primário de jogo através do qual vários serviços têm sido gamificados; constituem elementos que transmitem determinadas provas sociais e pistas (visuais e textuais) às quais estão associados significados - e.g. cumprimento de objetivos, comprometimento com os mesmos, sinalização de um estatuto e reputação (idem). A utilização deste mecanismo leva a bons resultados em contextos de jogos; no entanto, pouco se sabe sobre os seus efeitos no comportamento do utilizador em contextos onde os mesmos não estão predispostos para o jogo (idem).

Na segunda posição, Hamari, Sjöklint, and Ukkonen (2016) concluem que as motivações intrínsecas são um forte determinante da atitude, contrariamente às extrínsecas; todavia, no que respeita a intenções de uso contínuo, as motivações extrínsecas foram um preditor mais significativo, a par da diversão/prazer da atividade. Assim, apresentam a gamificação como exemplo para aumentar as motivações intrínsecas e refrear as 
motivações extrínsecas. Segundo os autores (idem., p.11), "o serviço deve ser prazeroso de usar, porque o prazer é um motivador importante".

Koivisto and Hamari (2014) estudaram os efeitos de género, idade e tempo de utilização de um serviço de exercício físico (Fitocracy) nos benefícios percebidos (sociais, hedónicos e utilitários) da gamificação. $\mathrm{O}$ principal objetivo deste tipo de serviços é motivar e apoiar o utilizador para aumentar a prática de exercício físico (propósito utilitário) através da adição de elementos hedónicos (caraterísticos dos jogos) à atividade: feedback, objetivos alcançáveis, progressão e encorajamento; pretende-se tornar a experiência de exercício físico mais divertida (Koivisto \& Hamari, 2014).

\section{Quadro V}

As 10 publicações científicas mais citadas.

\begin{tabular}{|c|c|c|c|c|}
\hline Título & Revista & Autores & Ano & Citações \\
\hline $\begin{array}{l}\text { Transforming homo economicus into } \\
\text { homo ludens: A field experiment on } \\
\text { gamification in a utilitarian peer-to-peer } \\
\text { trading service }\end{array}$ & $\begin{array}{l}\text { Electronic } \\
\text { Commerce } \\
\text { Research and } \\
\text { Applications }\end{array}$ & Hamari, Juho & 2013 & 108 \\
\hline $\begin{array}{l}\text { The sharing economy: Why people } \\
\text { participate in collaborative consumption }\end{array}$ & $\begin{array}{l}\text { Journal of the } \\
\text { Association for } \\
\text { Information Science } \\
\text { and Technology }\end{array}$ & $\begin{array}{l}\text { Hamari, } \\
\text { Sjoklint \& } \\
\text { Ukkonen. }\end{array}$ & 2016 & 88 \\
\hline $\begin{array}{l}\text { Demographic differences in perceived } \\
\text { benefits from gamification }\end{array}$ & $\begin{array}{l}\text { Computers in } \\
\text { Human Behavior }\end{array}$ & $\begin{array}{l}\text { Koivisto, } \\
\text { Jonna \& } \\
\text { Hamari, Juho }\end{array}$ & 2014 & 82 \\
\hline $\begin{array}{l}\text { "Working out for likes": An empirical } \\
\text { study on social influence in exercise } \\
\text { gamification }\end{array}$ & $\begin{array}{l}\text { Computers in } \\
\text { Human Behavior }\end{array}$ & $\begin{array}{l}\text { Hamari, Juho } \\
\text { \& Koivisto, } \\
\text { Jonna }\end{array}$ & 2015 & 36 \\
\hline $\begin{array}{l}\text { Gamification Design of IT-Based } \\
\text { Enhancing Services for Motivational } \\
\text { Support and Behavioral Change }\end{array}$ & $\begin{array}{l}\text { Business \& } \\
\text { Information } \\
\text { Systems } \\
\text { Engineering }\end{array}$ & $\begin{array}{l}\text { Blohm, Ivo \& } \\
\text { Leimeister, } \\
\text { Jan Marco }\end{array}$ & 2013 & 32 \\
\hline $\begin{array}{l}\text { Why do people use gamification } \\
\text { services? }\end{array}$ & $\begin{array}{l}\text { International } \\
\text { Journal of } \\
\text { Information } \\
\text { Management } \\
\end{array}$ & $\begin{array}{l}\text { Hamari, Juho } \\
\& \text { Koivisto, } \\
\text { Jonna }\end{array}$ & 2015 & 32 \\
\hline $\begin{array}{l}\text { Measuring flow in gamification: } \\
\text { Dispositional Flow Scale-2 }\end{array}$ & $\begin{array}{l}\text { Computers in } \\
\text { Human Behavior }\end{array}$ & $\begin{array}{l}\text { Hamari, Juho } \\
\text { \& Koivisto, } \\
\text { Jonna }\end{array}$ & 2014 & 24 \\
\hline $\begin{array}{l}\text { Playful training with augmented reality } \\
\text { games: case studies towards reality- } \\
\text { oriented system design }\end{array}$ & $\begin{array}{l}\text { Multimedia Tools } \\
\text { and Applications }\end{array}$ & $\begin{array}{l}\text { Yamabe, } \\
\text { Tetsuo; } \\
\text { Nakajima, } \\
\text { Tatsuo }\end{array}$ & 2013 & 23 \\
\hline $\begin{array}{l}\text { Gamified physical activation of young } \\
\text { men - a Multidisciplinary Population- } \\
\text { Based Randomized Controlled Trial } \\
\text { (MOPO study) }\end{array}$ & BMC Public Health & $\begin{array}{l}\text { Ahola, Riikka; } \\
\text { Pyky, Riitta; } \\
\text { Jamsa, Timo; } \\
\text { et al. }\end{array}$ & 2013 & 19 \\
\hline $\begin{array}{l}\text { Gamification-Based Incentive } \\
\text { Mechanism for Participatory Sensing }\end{array}$ & $\begin{array}{l}\text { International } \\
\text { Conference on } \\
\text { Pervasive } \\
\text { Computing and } \\
\text { Communications }\end{array}$ & $\begin{array}{l}\text { Ueyama, } \\
\text { Yoshitaka; } \\
\text { Tamai, } \\
\text { Morihiko; } \\
\text { Arakawa, } \\
\text { Yutaka; et al. }\end{array}$ & 2014 & 16 \\
\hline
\end{tabular}




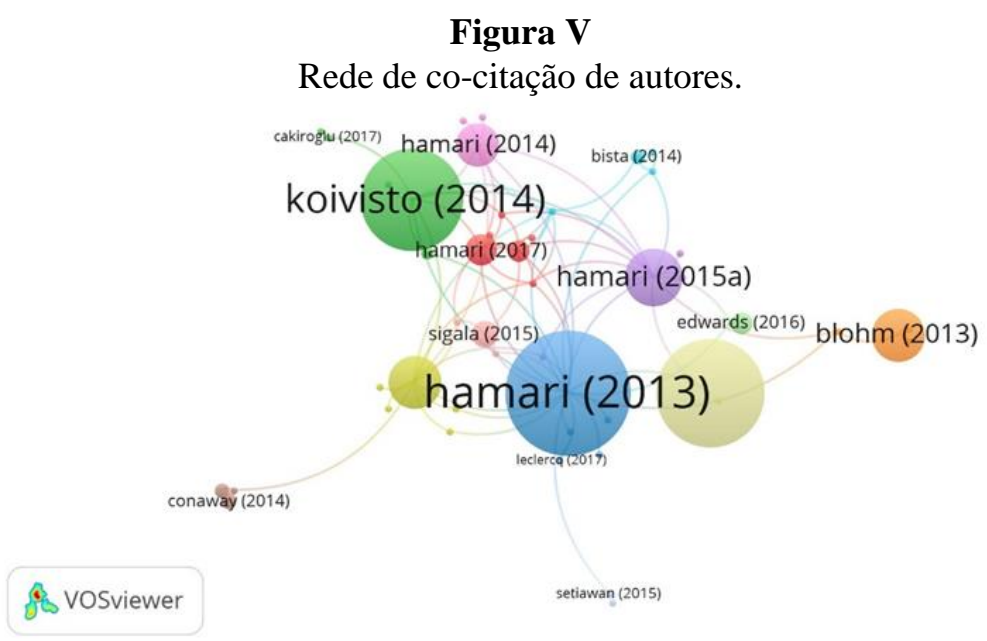

Ao estudar os efeitos de género, Koivisto and Hamari (2014) descobriram que as mulheres valorizam mais os aspetos sociais da gamificação, percecionam mais os benefícios da facilidade de uso da tecnologia e percecionam o exercício gamificado como mais divertido. Quanto aos efeitos da idade, concluíram (idem, p.183) que "quanto mais maduros são os utilizadores, menos experienciam a facilidade de uso" de serviços gamificados. Concluíram, também, que a diversão e a utilidade percebidas da gamificação tendem a diminuir ao longo da utilização do serviço. Poderão, portanto, existir efeitos de novidade da gamificação, visto que à medida que o utilizador continua a usar o serviço, o seu interesse e entusiasmo vai desvanecendo-se.

Hamari and Koivisto (2015b) (em quarto lugar) estudaram de que forma os aspetos sociais influenciam as pessoas a iniciar e manter comportamentos benéficos promovidos pela gamificação, especificamente no que respeita à prática de atividade física. Concluíram, (idem, p. 333) que "a influência social, o reconhecimento positivo e a reciprocidade afetam positivamente a disposição das pessoas para praticar exercício, bem como as suas atitudes e disposição para usar os serviços gamificados".

Hamari and Koivisto (2015a) (sexta posição) exploraram os benefícios que motivam as pessoas a usar serviços gamificados. Concluíram que a 
utilização e a aceitação da gamificação é fortemente motivada por benefícios utilitários e hedónicos. Já os benefícios sociais não estão associados às intenções para continuar a usar o serviço, mas influenciam significativa e positivamente a atitude relativamente ao sistema. Noutras palavras, "os aspetos hedónicos (motivadores intrínsecos) incentivam o uso efetivo, enquanto os aspetos sociais e utilitários (motivadores extrínsecos) afetam a atitude, a qual tem um efeito nas intenções de uso" (Hamari \& Koivisto, 2015a, p. 427).

Hamari and Koivisto (2014) focaram-se na análise psicométrica do instrumento Dispositional Flow Scale-2 (DFS-2) no contexto da gamificação do exercício físico. O referido instrumento mede o fluxo e é "usado para descrever a experiência ideal caraterizada por um estado de completa imersão e envolvimento na atividade" (Csíkszentmihályi, 1990, cit in Hamari \& Koivisto, 2014, p. 133). Hamari and Koivisto (2014) concluíram que a estrutura fatorial original de Jackson e Eklund (2002) é adequada à situação analisada. Referem que a gamificação e a disseminação dos vídeo jogos leva a que as pessoas se envolvam cada vez mais em atividades com potencial de induzir a um estado de fluxo. Em atividades quotidianas que exigem níveis elevados de persistência, resiliência e comprometimento (e.g. exercício físico, consumo sustentável) por parte dos indivíduos, entrar num estado de fluxo através da gamificação dessas atividades pode ter grandes vantagens.

Yamabe and Nakajima (2013) analisaram a gamificação aplicada ao contexto educativo, procurando implementar caraterísticas de jogo a ambientes de aprendizagem tradicionais. Embora a curta duração dos estudos tenha impedido resultados significativos e fiáveis, os autores referem que o aumento na interação física levou a experiências prazerosas para o utilizador, levando-o a direcionar maior atenção ao jogo e à atividade de aprendizagem - facto que normalmente não ocorre num processo de aprendizagem comum (o qual facilmente se torna aborrecido).

Efetivamente, "jogar é uma atividade humana altamente intelectual, sendo possível desenvolver capacidades e adquirir conhecimentos" (Yamabe \& Nakajima, 2013, p. 260). Se, por um lado, os jogos permitem a prática repetitiva sem sérios riscos, também acabam por motivar e entreter o utilizador, aumentando a probabilidade de melhores desempenhos. 
Ahola et al. (2013) focaram-se no potencial da gamificação no âmbito dos serviços de saúde, incentivo à atividade física e promoção de estilos de vida saudáveis, especificamente para jovens do sexo masculino.

Ueyama et al. (2014) estudaram de que forma a gamificação pode ser utilizada como mecanismo de incentivo para o participatory sensing (isto é, alcançar uma participação ativa dos utilizadores em processos de deteção de informação), para além dos tradicionais incentivos monetários. Neste caso, os utilizadores de telemóveis são aproveitados como sensores de recolha de informações nos pontos de interesse numa cidade, sem necessidade de sensores fixos (por exemplo, a monitorização do trânsito em tempo real). $\mathrm{O}$ mecanismo proposto pelos referidos autores "permite aos utilizadores obter recompensas monetárias como também satisfação (sentimento de realização) ao obter emblemas e prémios no ranking da gamificação" (Ueyama et al., 2014, p. 98). Os resultados confirmaram que, de facto, "o mecanismo de gamificação aumentou a probabilidade de participação de 53\% (sem gamificação) para 73\%” (idem, p. 98).

\subsection{Análise da co-citação das revistas científicas}

Quando dois itens (e.g. artigos, revistas, autores) estão citados simultaneamente numa lista de referência de um terceiro, eles têm uma relação de co-citação; nesta análise visa-se "revelar a relação e a estrutura de autores, artigos e revistas em áreas académicas" (Liao et al., 2018, p. 11). Concretamente, a análise da co-citação de revistas científicas é uma "forma eficaz de estudar a estrutura e as caraterísticas de um tópico, mas também revela a estrutura geral do tema e a caraterísticas da revista" (idem, 2018, p. 13).

Foi criada a rede de co-citação de revistas, com 31 pontos - Figura VI. A dimensão dos pontos representa a atividade da revista e o número de papers publicados; quanto menor a distância entre eles, maior a frequência de citação. Na Figura VI, cada cluster tem uma cor que indica o grupo ao qual pertence. Neste caso, as revistas estão divididas em três clusters. $\mathrm{O}$ verde inclui aquelas mais focadas na perspetiva da psicologia - e.g. Computers in Human Behavior (da Elsevier) - mas também no âmbito dos serviços de tecnologias de informação - e.g. MIS Quarterly (Minnesota). O cluster vermelho contém vários tipos de revistas e conferências que se centram numa perspetiva da educação, medicina, 
media, ciências computacionais e tecnologias emergentes. O cluster azul representa maioritariamente revistas no âmbito do marketing e gestão de negócios.

\section{Figura VI}

Rede de co-citação de revistas científicas relacionadas com as publicações de gamificação e serviços.

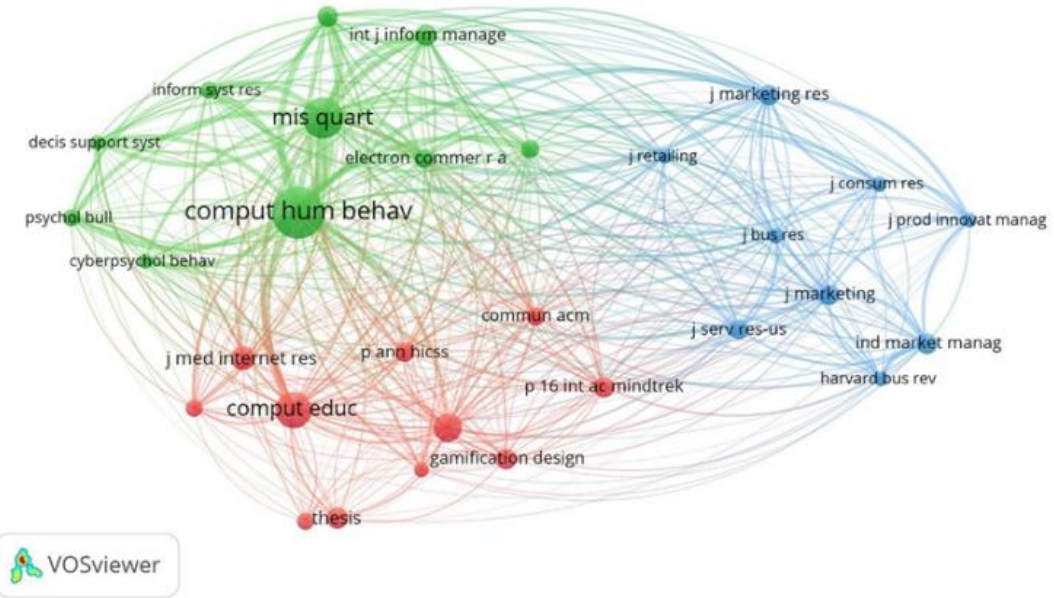

O Quadro VI ilustra a distribuição das principais fontes utilizadas no estudo da gamificação e serviços. Destaca-se a revista Computers in Human Behavior, tendo sido aquela com maior número de citações (170) e maior Total Link Strength (3289). 


\section{Quadro VI}

As 10 revistas científicas chave

\begin{tabular}{llcc}
\hline \multicolumn{1}{c}{ Fonte } & \multicolumn{1}{c}{ Tópico } & Frequência & Total Link Strength \\
\hline $\begin{array}{l}\text { comput human } \\
\text { behav }\end{array}$ & Computers in Human Behavior & 170 & 3289 \\
mis quart & MIS Quarterly & 110 & 2721 \\
comput educ & Computers \& Education & 94 & 1042 \\
p 15 int ac mindtrek & $\begin{array}{l}\text { 15th International Academic MindTrek } \\
\text { Conference }\end{array}$ & 61 & 680 \\
j med internet res & Journal of Medical Internet Research & 45 & 303 \\
thesis & & 40 & 163 \\
int j inform manage & International Journal of Information & 39 & 1004 \\
& Management & & \\
inform manage- & Information \& Management & 34 & 997 \\
amster & Journal of Marketing Research & 34 & 889 \\
j marketing res & Journal of Marketing & 34 & 739
\end{tabular}

\section{CONCLUSÃO}

O objetivo da Scoping Review foi perceber o estado atual do estudo científico no âmbito da gamificação e dos serviços.

Com base nas análises levadas a cabo foi possível verificar que o estado atual do estudo científico desta área se encontra numa fase consideravelmente inicial e embrionária, visto que apenas a partir do ano 2011 há registo de artigos alusivos ao tema na base de dados consultada. De outro modo, identificou-se também o interesse cada vez maior relativamente a este domínio, na medida em que os indicadores vão no sentido de uma tendência de crescimento e expansão progressiva na quantidade de publicações científicas. Esta conclusão vai ao encontro daquilo que também Hamari and Koivisto (2015b, p. 333) afirmam - "a quantidade de investigação sobre o tópico está a aumentar rapidamente.

Em termos dos serviços nos quais a gamificação tem sido mais estudada e implementada, os resultados revelaram claramente o destaque dos serviços de saúde e dos serviços de educação. De forma geral, a implementação da gamificação nos serviços passa por ajudar e motivar os utilizadores para adotar determinados comportamentos benéficos. Para atingir tal finalidade, os autores realçam a importância do apoio e benefícios sociais decorrentes da interação com outros utilizadores para 
continuar o comportamento suportado pelo sistema (Hamari \& Koivisto, 2015b).

Numa outra perspetiva, e através da análise de citações realizada, descobriu-se que o país que tem vindo a assumir um papel central e de destaque no estudo da gamificação e serviços é a Finlândia. Ao nível das organizações, verificou-se que aquelas com papéis centrais no estudo destes tópicos são também finlandesas.

Por último, foi possível identificar os três autores centrais no estudo da gamificação e serviços, são eles Hamari J., Koivisto J. e Nakajima T.

\section{Limitações}

Tendo em conta o número reduzido de publicações científicas, bem como o facto deste tópico de estudo - gamificação e serviços - ser ainda muito recente, leva a que as análises realizadas sejam pouco abrangentes e profundas de forma a ser possível identificar tendências e padrões claros e significativos nos dados; ou seja, as conclusões retiradas são de âmbito limitado. Por exemplo, não foi possível detetar padrões de palavras-chave ao longo do tempo, obstaculizando a identificação de tendências evidentes.

\section{Investigações Futuras}

Sendo este um tópico ainda muito recente faz com que haja uma grande margem para investigação futura. Tendo em conta a grande abrangência inerente à definição daquilo que são os serviços na sociedade contemporânea parece haver aqui um campo fértil dentro do qual ainda há muito por saber.

Encontramo-nos hoje numa era onde a transformação digital desempenha um papel cada vez mais central, trazendo consigo drásticas mudanças a vários níveis - na vida quotidiana das pessoas, nas relações interpessoais, no contexto em que nos inserimos, nos negócios que surgem (e na forma como se realizam), nos problemas que enfrentamos (e sua resolução), na forma como vemos o mundo e como reagimos ao que nele ocorre. Ora, inerente a esta transformação digital vem uma miríade de oportunidades, mas também desafios. Assim, tendo em conta o contexto atual, poderá ser interessante e útil compreender qual o papel da gamificação - enquanto abordagem tecnológica inovadora -, bem como 
realizar esforços de modo a identificar possíveis aplicações nas organizações que, neste momento, estão a passar por esse processo de transformação digital.

\section{FONTES E BIBLIOGRAFIA}

Ahola, R., et al. (2013). Gamified physical activation of young men-a Multidisciplinary Population-Based Randomized Controlled Trial (MOPO study). BMC public health, 13(1), 32.

Almeida, S. L. de, Capucho, F., \& Ribeiro, P. (2017). Vamos Comunicar? Gestão e Desenvolvimento, 25, 27-53.

Arksey, H., \& O'Malley, L. (2005). Scoping studies: towards a methodological framework. International journal of social research methodology, 8(1), 19-32.

EBSCO. (2018). EBSCOhost Research Databases. Disponível em: http://www.biblioteca.porto.ucp.pt

Hamari, J. (2013). Transforming homo economicus into homo ludens: A field experiment on gamification in a utilitarian peer-to-peer trading service. Electronic commerce research and applications, 12(4), 236245.

Hamari, J., \& Koivisto, J. (2014). Measuring flow in gamification: dispositional flow scale-2. Computers in Human Behavior, 40, 133143.

Hamari, J., \& Koivisto, J. (2015a). Why do people use gamification services? International Journal of Information Management, 35(4), 419-431.

Hamari, J., \& Koivisto, J. (2015b). "Working out for likes": An empirical study on social influence in exercise gamification. Computers in Human Behavior, 50, 333-347.

Hamari, J., Sjöklint, M., \& Ukkonen, A. (2016). The sharing economy: Why people participate in collaborative consumption. Journal of the Association for Information Science and Technology, 67(9), 20472059.

Koivisto, J., \& Hamari, J. (2014). Demographic differences in perceived benefits from gamification. Computers in Human Behavior, 35, 179188. 
Liao, H., Tang, M., Luo, L., Li, C., Chiclana, F., \& Zeng, X.-J. (2018). A Bibliometric Analysis and Visualization of Medical Big Data Research. Sustainability, 10(1), 166.

Lopes, C. P., \& Andrade, A. M. V. De. (2008). Simuladores e o Ensino da Economia e da Gestão no Ensino Superior em Portugal. In M. P. Cota (Ed.) (3.a, Vol. I, pp. 129-141). Ourense: LibroTex.

Lopes, S., Costa, M. T., Fernández-Llimós, F., Amante, M. J., \& Lopes, P. F. (2012). A Bibliometria e a Avaliação da Produção Científica: indicadores e ferramentas. Paper presented at the Actas do congresso Nacional de bibliotecários, arquivistas e documentalistas.

Tahamtan, I., Afshar, A. S., \& Ahamdzadeh, K. (2016). Factors affecting number of citations: a comprehensive review of the literature. Scientometrics, 107(3), 1195-1225.

Ueyama, Y., Tamai, M., Arakawa, Y., \& Yasumoto, K. (2014). Gamification-based incentive mechanism for participatory sensing. Paper presented at the Pervasive Computing and Communications Workshops (PERCOM Workshops), 2014 IEEE International Conference on.

Yamabe, T., \& Nakajima, T. (2013). Playful training with augmented reality games: case studies towards reality-oriented system design. Multimedia Tools and Applications, 62(1), 259-286. 